\title{
Utilização dos aparelhos digitais em crianças com idade entre os 12 meses e os 5 anos
}

Nuno Miguel Parente, ${ }^{1}$ Ana Elisabete Costa, ${ }^{2}$ Ana Pinho Matos, ${ }^{2}$ Carla Barbosa Duarte, ${ }^{2}$ Carina Freitas, $^{3}$ Diana Mota, ${ }^{4}$ Mário Pedro Martins, ${ }^{5}$ Miguel Santos Martins ${ }^{6}$

\section{RESUMO}

Objetivo: Caracterizar a utilização dos aparelhos digitais em idade pré-escolar e comparar com as recomendações da Academia Americana de Pediatria e OMS. Testar associações entre a utilização dos aparelhos digitais pelas crianças e determinadas características dos pais e do seu uso.

Tipo de estudo: Estudo transversal, descritivo e analítico.

Local: Unidades de Saúde Familiar (USF) da zona norte de Portugal.

População: Utentes, com idade entre os 12 meses e os cinco anos inclusive, de seis USF.

Métodos: Questionário anónimo aplicado a uma amostra de pais que acompanharam a criança à consulta. Incluiu variáveis demográficas e perguntas relacionadas com a utilização dos aparelhos digitais pela criança e pais.

Resultados: Obtiveram-se 342 questionários. A televisão e o smartphone foram os aparelhos mais presentes nas casas e os mais frequentemente usados pelas crianças. O início do seu uso deu-se antes dos 18 meses em 74,7\% para a televisão, 23,4\% para o smartphone e 16,3\% para o tablet. Em 65,9\% e $24 \%$ houve uma utilização diária para a televisão e smartphone, respetivamente. A utilização dos aparelhos aconteceu em $19 \%$ nos 60 minutos antes do sono e o «uso às refeições» foi o motivo mais reportado. Nas crianças com idade < 24 meses $88-93 \%$ demonstraram utilizar aparelhos digitais, enquanto nas crianças idade > 24 meses 70-74\% tinham um tempo de ecrã > 1h/dia. Verificou-se associação do tempo de ecrã das crianças com o tempo de ecrã dos pais, perceção dos pais relativamente ao TEE da criança e número de aparelhos digitais no quarto.

Conclusões: Regista-se um início muito precoce, antes dos 18 meses, para o uso destas tecnologias, que é abusivo, preocupante e fora das normas de orientação atuais. É importante abordar este assunto desde cedo nas consultas, sensibilizando os pais para outros tipos de atividade e estímulo.

Palavras-chave: Tempo de exposição de ecrã; Normas de orientação clínica; Comportamento; Crianças; Pais.

\section{INTRODUÇÃO}

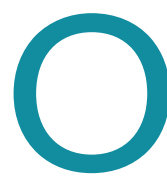

s aparelhos digitais encontram-se amplamente difundidos na sociedade atual, fazendo parte das rotinas diárias de qualquer família. Se, por um lado, esta era tecnológica se assume como um horizonte sem limites ou barreiras informativas, por outro lado, pode impulsionar uma exposição não regrada e excessiva a conteúdos inadequados, que condicionam o desenvolvimento da

1. USF Viver Mais, ACeS Maia/Valongo.

2. USF Salvador Lordelo, ACeS Vale do Sousa Sul.

3. USF Terras de Souza, ACeS Vale do Sousa Sul.

4. USF Freamunde, ACeS Vale do Sousa Norte.

5. USF Tempo de Cuidar, ACeS Vale do Sousa Sul.

6. USF Calâmbriga, ACeS Aveiro Norte. criança e acarretam potenciais implicações negativas, nomeadamente nos hábitos alimentares, de sono, na interação social e no aproveitamento escolar. ${ }^{1-6}$

Considerando estes potenciais efeitos negativos, a utilização adequada dos aparelhos digitais pelas crianças surge como um desafio a alcançar. Neste contexto, várias sociedades científicas têm desenvolvido recomendações, cujo objetivo é orientar pais, educadores e profissionais de saúde a diminuírem o uso abusivo destas tecnologias. $^{7-9}$

Neste âmbito, em 2016, a Academia Americana de Pediatria (AAP) elaborou recomendações acerca do tempo de exposição de ecrã (TEE) em crianças dos zero aos cinco anos, período considerado crítico para o 
neurodesenvolvimento, durante o qual se estabelecem relações de vinculação seguras e comportamentos saudáveis. ${ }^{7}$

Segundo essa entidade, a utilização de aparelhos digitais nas crianças antes dos 24 meses de idade não é recomendada, uma vez que estas crianças necessitam de interagir com os cuidadores e explorar o meio que os rodeia para adquirirem capacidades cognitivas, emocionais, motoras e de linguagem. Além disso, a imaturidade simbólica, característica desta faixa etária, não permite distinguir a fantasia apresentada da vida real. Por sua vez, dos dois aos cinco anos de idade, a AAP recomenda que o TEE seja limitado a uma hora por dia, com conteúdos adequados à fase de desenvolvimento da criança e sob supervisão dos adultos. ${ }^{6-7,10}$

Mais recentemente, em 2019, a Organização Mundial da Saúde (OMS) também elaborou guidelines neste âmbito, cujas recomendações são idênticas às preconizadas pela AAP ${ }^{9}$

O médico e enfermeiro de família, pelo contacto privilegiado que têm com as crianças e seus cuidadores, surgem como um importante interlocutor desta mensagem com as consultas de vigilância de saúde infantil e juvenil (SIJ), constituindo uma excelente oportunidade para a sua divulgação.

O presente trabalho tem como objetivo caracterizar a utilização dos aparelhos digitais numa amostra de crianças com idades entre os 12 meses e os cinco anos, inclusive, e comparar essa utilização com as recomendações da AAP e da OMS.

\section{MÉTODOS}

Foi conduzido um estudo transversal, descritivo e analítico, entre 1 de abril de 2018 e 1 de agosto de 2019, em seis Unidades de Saúde Familiar (USF) da zona norte de Portugal, pertencentes a quatro Agrupamentos de Centros de Saúde (ACeS). Os autores elaboraram um questionário de autopreenchimento, adaptado de bibliografia já publicada, anónimo, que foi aplicado aos pais das crianças..$^{1-6}$

O questionário incluiu três partes, a primeira associada a dados demográficos, a segunda à utilização dos aparelhos digitais pelas crianças e a terceira à utilização dos aparelhos digitais pelos pais (Anexo 1). As variáveis estudadas foram: idade, género, cuidador principal, idade dos pais, escolaridade dos pais, responsá- vel pelo preenchimento do questionário (parte I); aparelhos digitais presentes em casa, aparelhos digitais de uso habitual, início de uso de aparelhos digitais, frequência do uso de aparelhos digitais, TEE em dia semanal (horas e minutos), TEE em fim-de-semana, situações de uso dos aparelhos digitais, aparelhos digitais presentes/utilizados no quarto da criança, número de aparelhos digitais presentes/utilizados no quarto da criança (variável dummy), frequência do uso não-acompanhado, classificação do uso de aparelhos digitais, abordagem por profissional de saúde relativa ao uso de aparelhos digitais, vontade em ter abordagem pelo profissional de saúde (parte II); aparelhos digitais de uso habitual pelos pais, frequência do uso de aparelhos digitais pelos pais, TEE em dia semanal pelos pais, TEE em fim-de-semana pelos pais, frequência do uso de aparelhos digitais às refeições pelos pais, aparelhos digitais presentes/utilizados no quarto dos pais, número de aparelhos digitais presentes/utilizados no quarto dos pais (variável dummy) (parte III).

A população foi constituída por utentes com idade entre os 12 meses e os cinco anos, inclusive, inscritos nas seis USF em estudo, com uma consulta de SIJ no período do estudo definido para recolha de dados, acompanhadas pelo menos por um dos pais. Excluíram-se os casos em que os cuidadores foram incapazes de compreender ou comunicar linguagem portuguesa, oral e escrita, ou comunicar e em que o questionário não foi preenchido na totalidade. A população em estudo totalizou 3.063 utentes. Foi selecionada uma amostra de conveniência com base nos utentes que, aquando da efetivação da inscrição para consulta de SIJ, foram convidados a participar no estudo pelo secretariado clínico. O secretariado clínico das USF envolvidas foi responsável por obter uma listagem dos utentes com o intervalo de idades definido através do programa SINUS $®$ e entregar o questionário aos pais, utilizando um guião elaborado pelos investigadores. De forma a existir uniformização no convite pelo secretariado clínico foi realizada uma sessão de formação prévia com os investigadores. No caso de aceitação por parte dos pais foi entregue o questionário, que incluía em preâmbulo a explicação do propósito do estudo e consentimento informado. Os questionários foram recolhidos em caixa fechada e selada para o efeito.

O tamanho amostral foi determinado considerando um intervalo de confiança de 95\% e um nível de 


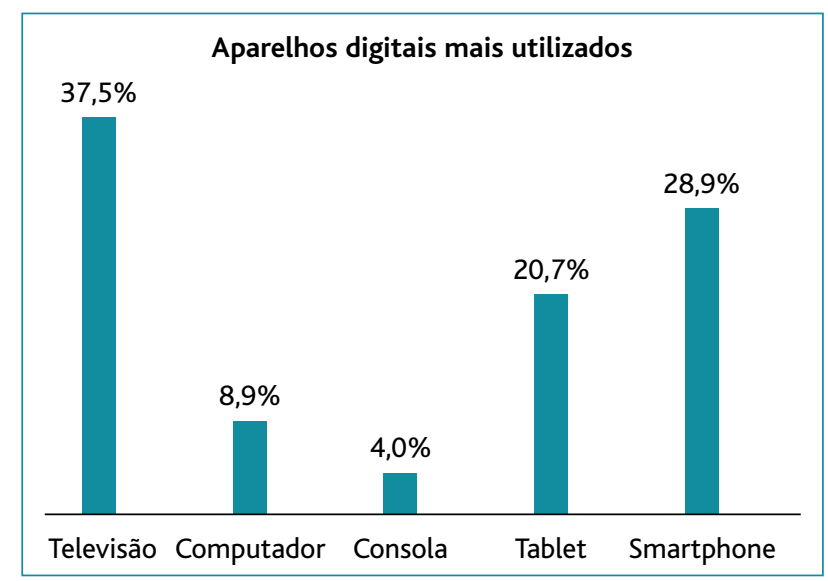

Figura 1. Aparelhos digitais mais utilizados pelas crianças. Legenda: \% = Percentagem.

precisão de $5 \%$, perfazendo um total de 341 participantes. Considerando o ajuste para perda de amostra de aproximadamente $10 \%$ assumiu-se um total de 380 participantes como tamanho amostral final.

Os dados foram submetidos a uma análise estatística com recurso aos programas Microsoft Excel $2010^{\circledR} \mathrm{e}$ SPSS $^{\circledast}$ v. (64 Bit) 23, em computadores pessoais dos autores, em pastas protegidas por password. Foram avaliadas associações entre o TEE da criança à semana/fim de semana e outras variáveis independentes, utilizando a correlação de Spearman, o teste de Mann-Whitney e o teste ANOVA. Foi também testada a associação do TEE durante as refeições entre pais e crianças recorrendo ao teste Qui-quadrado.

Foram utilizadas as linhas de orientação Strengthening the Reporting of Observational Studies in Epidemiology (STROBE) para a elaboração do presente artigo. ${ }^{11}$

\section{TABELA 1. Idade de início de utilização dos aparelhos digitais.}

\begin{tabular}{l|c|c|c|c|} 
Aparelho digital & $<\mathbf{1 8}$ Meses & $\mathbf{1 8 - 2 4}$ Meses & $>\mathbf{2 4}$ Meses & Nunca \\
\hline Televisão & $74,7 \%$ & $17,5 \%$ & $5,0 \%$ & $2,8 \%$ \\
\hline Tablet & $16,3 \%$ & $22,8 \%$ & $29,8 \%$ & $31,1 \%$ \\
\hline Smartphone & $23,4 \%$ & $28,4 \%$ & $28,9 \%$ & $19,3 \%$ \\
\hline Consola & $0 \%$ & $13,1 \%$ & $15,9 \%$ & $71,0 \%$ \\
\hline Computador & $4,0 \%$ & $5,5 \%$ & $14,0 \%$ & $76,5 \%$ \\
\hline
\end{tabular}

Legenda: $\%=$ Percentagem .
A realização deste estudo foi aprovada pelos ACeS das USF em estudo e mereceu parecer favorável por parte da comissão de ética da Administração Regional de Saúde do Norte.

\section{RESULTADOS}

Foram incluídos 342 utentes, correspondente a uma taxa de participação de $90 \%$. A população teve um predomínio do sexo masculino $(52,4 \%)$ e a seguinte distribuição etária: $20,3 \%$ (< 18 meses), 21,8\% (18-24 meses) e $57,9 \%$ (> 24 meses).

Relativamente à idade dos pais, $2,1 \%$ e 1,2\% das mães e pais tinham menos de 19 anos, respetivamente, e as faixas etárias predominantes foram a de «30-35 anos» nas mães (39,5\%) e «idade superior a 35 anos» nos pais $(52,9 \%)$. Quanto à escolaridade parental, a frequência do ensino secundário $(32,4 \%)$ nas mães e do $3^{\circ}$ ciclo do ensino básico $(32,0 \%)$ nos pais foram os mais representativos. De referir que, da amostra dos pais, $10,8 \%$ tinha como escolaridade o $1^{\circ}$ ciclo do ensino básico e que $51,9 \%$ o ensino superior.

No que concerne aos cuidados prestados à criança, a maioria do tempo em que está acordada é passado no infantário $(38,6 \%)$ ou com os pais $(31,6 \%)$, sendo que os avós e a ama corresponderam a $20,1 \%$ e $9,7 \%$, respetivamente. A maioria dos questionários $(70 \%)$ foram preenchidos por mães.

Na parte II, os aparelhos digitais mais comuns nas casas das famílias inquiridas foram a televisão $(26,1 \%)$ e o smartphone (25,3\%). No que diz respeito ao uso, os aparelhos digitais mais utilizados pelas crianças foram também a televisão (37,5\%) e o smartphone (28,9\%) (Figura 1). Em relação à idade de início de uso, $74,7 \%$ das crianças começaram a ver televisão antes dos 18 meses. O tablet e o smartphone foram predominantemente iniciados após os 24 meses em $29,8 \%$ e $28,9 \%$ dos casos, respetivamente, enquanto $71 \%$ e $76,5 \%$ das crianças nunca utilizaram, respetivamente, consola ou computador (Tabela 1).

Quanto à frequência de utilização, a televisão foi usada 5 a 7 vezes/semana em $65,9 \%$, o tablet e o smartphone foram usados predominantemente 3 a 4 vezes/semana em $20,8 \%$ e $32,6 \%$, respetivamente. A consola foi utilizada principalmente $1 \mathrm{a}$ 


\section{Frequência de situações específicas de uso}

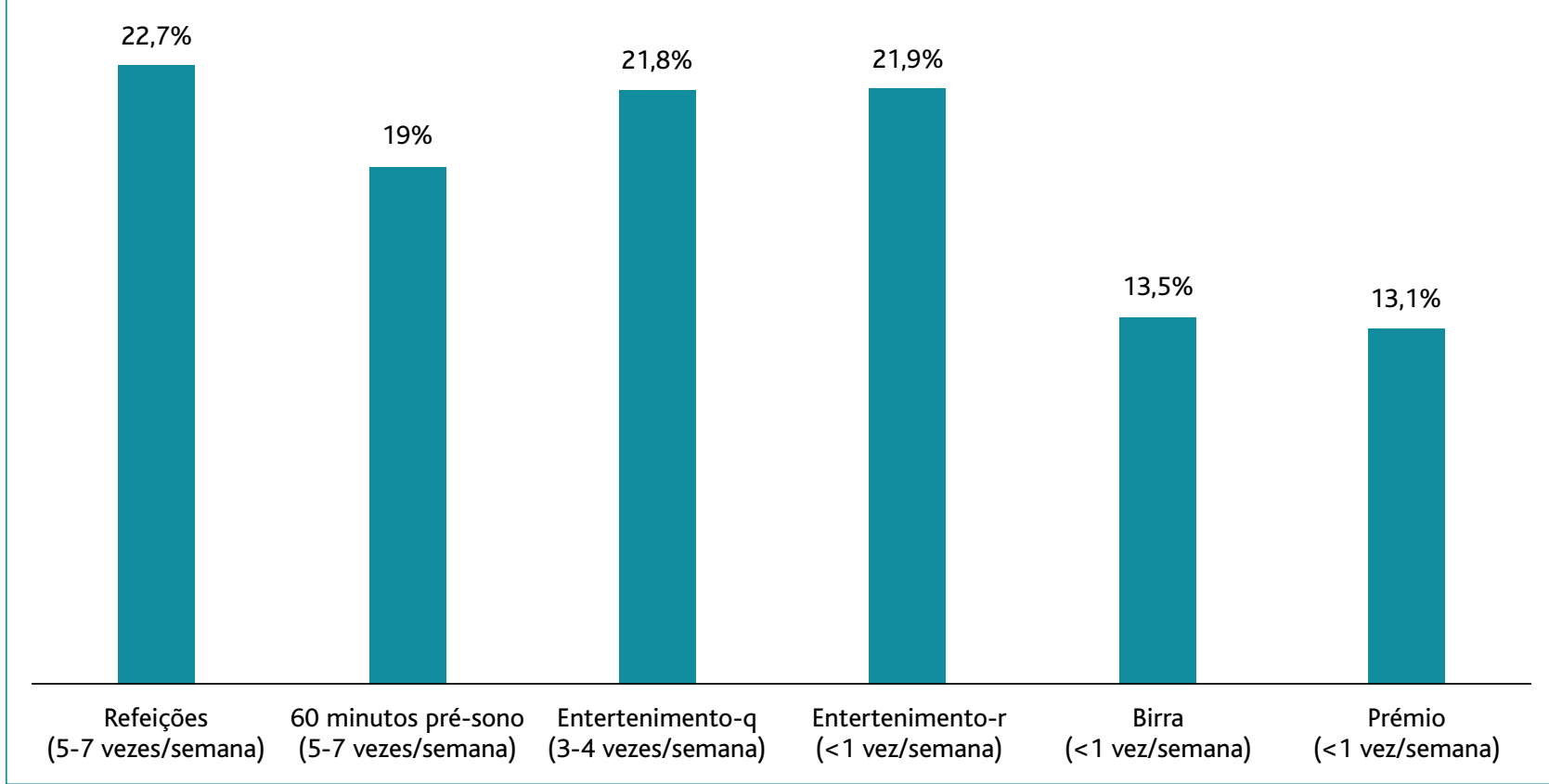

Figura 2. Frequência de situações específicas de utilização de aparelhos digitais.

Legenda: Entretenimento- $q$ = Entretenimento durante tarefas de quotidiano dos pais; Entretenimento-r = Entretenimento durante reuniões dos pais com amigos/familiares; \% = Percentagem.

2 vezes/semana em $8,2 \%$, com aproximadamente $74 \%$ a reportar «nunca usou». As médias do TEE foram de 1,66 horas (100 minutos) durante a semana e de 2,04 horas (122 minutos) ao fim de semana.

No que se refere à distribuição pela idade, aproximadamente $70-74 \%$ das crianças com $>24$ meses utilizam mais do que uma hora por dia, enquanto $88-93 \%$ das crianças com $<24$ meses utilizam aparelhos digitais nalgum momento do quotidiano.

No que concerne à utilização de aparelhos digitais em situações específicas do quotidiano (Figura 2), a resposta «nunca usou» foi a mais prevalente transversalmente. No entanto, verificou-se que a utilização de aparelhos digitais ocorreu em $22,7 \%$ durante os períodos das refeições, 5 a 7 vezes/semana; 19,0\% durante os 60 minutos antes do sono, 5 a 7 vezes/semana; $21,8 \%$ para entretenimento durante as tarefas do quotidiano dos pais, 3 a 4 vezes/semana; 21,9\% para entretenimento durante as reuniões com família/amigos, $<1$ vez/semana; $13,5 \%$ para distração durante uma birra,
$<1 \mathrm{vez} /$ semana; e $13,1 \%$ para premiar a criança, $<1$ vez/semana.

Quanto à existência/uso de aparelhos digitais no quarto da criança, a televisão, o tablet e o smartphone foram os mais reportados em $41 \%, 27 \%$ e $21 \%$, respetivamente. Para além disso, há disponibilização de um aparelho digital no quarto da criança em $39,6 \%$ das casas e dois em $12,1 \%$.

Relativamente à utilização de aparelhos digitais de forma autónoma pela criança (Figura 3), a resposta «nunca usou» foi a mais frequentemente reportada para qualquer aparelho. Constatou-se que, na frequência de 5 a 7 vezes/semana, a televisão foi usada pela criança quanto está sozinha em $25,2 \%$ e o smartphone em $12,9 \%$, enquanto na frequência de 1 a 2 vezes/semana o tablet foi usado pela criança quanto está sozinha em $16,8 \%$ dos casos.

Quanto à opinião dos pais sobre o uso destes aparelhos pelos seus filhos, 49,5\% classificaram-no como «adequado», $26,8 \%$ como «reduzido» e $23,6 \%$ como 


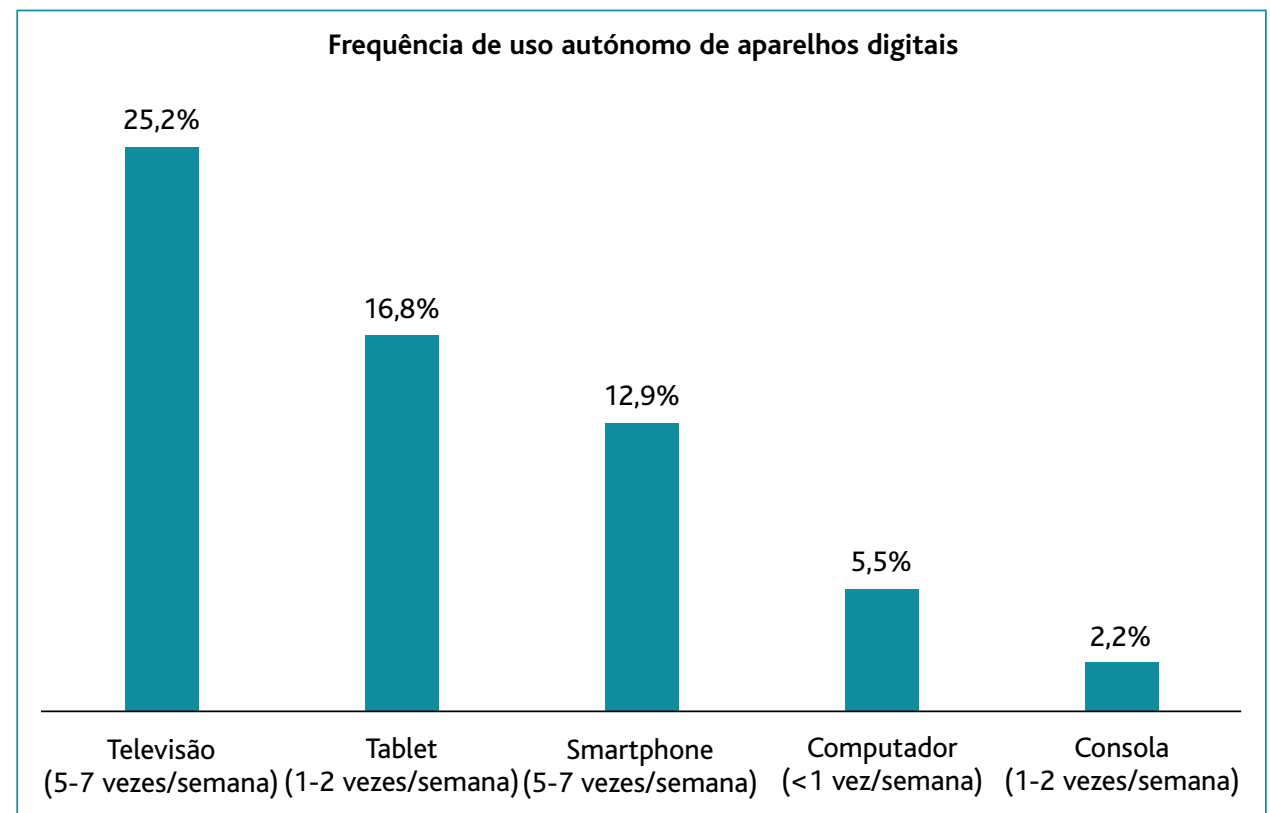

Figura 3. Frequência de uso autónomo de aparelhos digitais pela criança. Legenda: \% = Percentagem.

«exagerado». Foi reportado que em $37,4 \%$ e $35,2 \%$ dos casos tinha existido informação prévia sobre a utilização de aparelhos digitais pelas crianças proveniente do médico de família e enfermeiro de família, respetivamente. Cerca de $76 \%$ dos inquiridos mostraram vontade em ver este assunto discutido e abordado durante as consultas de SIJ.

Na parte III, os aparelhos digitais mais utilizados pelos pais foram a televisão (34,0\%), o smartphone (21,7\%) e o computador $(20,4 \%)$. Relativamente à sua frequência de uso, os aparelhos digitais foram utilizados predominantemente 5 a 7 vezes/semana, em que o smartphone totalizou 93,5\% dos casos, a televisão 72,6\% e o tablet $24,9 \%$. As médias do TEE foram de 4,40 horas (264 minutos) durante a semana e de 3,42 horas (205 minutos) ao fim de semana. No que concerne ao uso destes aparelhos às refeições, $37,6 \%$ reportou «nunca usou" e $21,2 \%$ e 10,9\% utilizaram 5 a 7 vezes/semana e 3 a 4 vezes/semana, respetivamente.

Quanto à existência/uso de aparelhos digitais no quarto dos pais, a televisão, o smartphone e o tablet foram os mais reportados em $43,3 \%, 37,1 \%$ e $10,8 \%$, respetivamente.

Verificou-se que o TEE das crianças durante a semana e ao fim de semana se correlacionou com o TEE dos pais durante a semana e ao fim de semana; perceção dos pais de utilização dos aparelhos digitais pelos filhos e o número de aparelhos digitais presentes no quarto da criança Tabela 2. Foi também verificada a existência de associação estatisticamente significativa entre o TEE às refeições dos pais e crianças $(p<0,0001)$.

\section{DISCUSSÃO}

Os dados apresentados evidenciam hábitos inadequados face às recomendações constantes na literatura. De acordo com as guidelines da AAP e OMS, às crianças até aos dois anos de idade não é aconselhada a utilização de aparelhos digitais e, entre os dois e os cinco anos, recomenda-se que o TEE seja limitado a uma hora por dia e a conteúdos adequados à fase de desenvolvimento da criança, sempre sob supervisão dos adultos. ${ }^{7,9}$

Quanto a um dos objetivos do trabalho, apenas 7\% das crianças com idade inferior a 24 meses não está exposta à utilização de aparelhos digitais, durante a semana ( $\nu$ s 93\% de utilizadores), aumentando o valor para cerca de $12 \%$ ao fim de semana ( $\nu s 88 \%$ de utilizadores). Nestes, a média do TEE durante a semana foi de 1,24 horas $( \pm 1,51)$ e ao fim de semana de 1,21 horas $( \pm 1,31)$. Relativamente às crianças com idade entre os dois e os cinco anos de idade, apenas cerca de $30,4 \%$ não ultrapassa, durante a semana, uma hora de utilização recomendada para esta faixa etária. Já ao fim de semana, o valor diminui para $26,3 \%$, refletindo uma maior utilização. Nestes, a média de TEE durante a semana foi de 1,97 horas $( \pm 1,67)$ e ao fim de semana de 2,67 horas $( \pm 2,13)$. Estes dados são condizentes com a evidência disponível, que nos mostra que a não conformidade das recomendações acima referidas acontece em $86 \%$ das crianças com 18 meses no Japão e em $90 \%$ das crianças com 24 meses nos Estados Unidos da América. ${ }^{3,12} \mathrm{O}$ facto das crianças com 


\begin{tabular}{|c|c|c|c|c|c|}
\hline \multicolumn{6}{|c|}{ Associação do tempo de ecrã com diferentes variáveis } \\
\hline \multirow{2}{*}{$\begin{array}{l}\text { Variáveis } \\
\text { Idade (Mãe) }\end{array}$} & \multicolumn{2}{|c|}{ TEE em horas (semana) } & \multicolumn{2}{|c|}{ TEE em horas (fim-de-semana) } & \multirow[t]{7}{*}{ Teste estatístico } \\
\hline & (média, DP) & & (média, DP) & & \\
\hline$\leq 19$ anos & $2,95( \pm 3,32)$ & & $3,49( \pm 2,64)$ & & \\
\hline 20-24 anos & $1,36( \pm 1,14)$ & & $1,57( \pm 1,51)$ & & \\
\hline 25-29 anos & $1,83( \pm 1,70)$ & $p=0,261$ & $2,15( \pm 1,87)$ & $p=0,293$ & \\
\hline 30-34 anos & $1,40( \pm 1,31)$ & & $1,94( \pm 1,97)$ & & \\
\hline$\geq 35$ anos & $1,89( \pm 1,93)$ & & $2,14( \pm 2,02)$ & & \\
\hline \multicolumn{6}{|l|}{ Idade (Pai) } \\
\hline$\leq 19$ anos & $2,50( \pm 2,12)$ & & $4,00( \pm 2,83)$ & & \\
\hline 20-24 anos & $1,44( \pm 1,14)$ & & $1,45( \pm 1,58)$ & & \\
\hline $25-29$ anos & $1,75( \pm 2,08)$ & $p=0,923$ & $2,13( \pm 1,97)$ & $p=0,476$ & \\
\hline 30-34 anos & $1,61( \pm 1,36)$ & & $2,15( \pm 2,01)$ & & \\
\hline$\geq 35$ anos & $1,67( \pm 1,73)$ & & $1,96( \pm 1,94)$ & & \\
\hline \multicolumn{6}{|c|}{ Escolaridade (Mãe) } \\
\hline $1-4^{\circ}$ ano & $1,81( \pm 1,55)$ & & $2,62( \pm 2,51)$ & & ANOVA \\
\hline $5-6^{\circ}$ ano & $1,61( \pm 2,18)$ & & $1,99( \pm 2,30)$ & & \\
\hline $7-9^{\circ}$ ano & $1,71( \pm 1,70)$ & $p=0,929$ & $1,70( \pm 1,52)$ & $p=0,09$ & \\
\hline $10-12^{\circ}$ ano & $1,55( \pm 1,80)$ & & $1,85( \pm 2,06)$ & & \\
\hline$>12^{\circ}$ ano & $1,73( \pm 1,28)$ & & $2,47( \pm 1,91)$ & & \\
\hline \multicolumn{6}{|c|}{ Escolaridade (Pai) } \\
\hline $1-4^{\circ}$ ano & $1,86( \pm 1,93)$ & & $2,38( \pm 2,60)$ & & \\
\hline $5-6^{\circ}$ ano & $1,69( \pm 2,03)$ & & $1,94( \pm 1,87)$ & & \\
\hline $7-9^{\circ}$ ano & $1,55( \pm 1,68)$ & $p=0,671$ & $1,63( \pm 1,62)$ & $p=0,027^{*}$ & \\
\hline $10-12^{\circ}$ ano & $1,57( \pm 1,59)$ & & $2,03( \pm 1,98)$ & & \\
\hline$>12^{\circ}$ ano & $1,89( \pm 1,40)$ & & $2,67( \pm 2,14)$ & & \\
\hline \multicolumn{6}{|c|}{ Perceção do tempo de ecrã do filho } \\
\hline Reduzido & $0,94( \pm 1,32)$ & $p<0,0001^{*}$ & $1,02( \pm 1,08)$ & $p<0,0001^{*}$ & \\
\hline Adequado & $1,80( \pm 1,57)$ & & $2,13( \pm 1,89)$ & & \\
\hline Exagerado & $2,30( \pm 1,86)$ & & $3,14( \pm 2,24)$ & & \\
\hline
\end{tabular}

idade inferior a 24 meses apresentarem menor taxa de utilização ao fim de semana poderá ser justificado pela maior disponibilidade dos pais ou outros cuidadores para atividades que não envolvem exposição de ecrã. De facto, Yeung e colaboradores demonstraram que o pai, considerando a sua remuneração e horário de trabalho nas famílias americanas, dispõe de 2,5h para atividades com os filhos durante a semana comparativamente com 6,5h ao fim de semana. ${ }^{13} \mathrm{O}$ mesmo já não se verifica para o grupo de crianças acima dos 24 meses, muito prova- velmente pela maior permissividade dos pais e cuidadores, mas também pela maior autonomia e solicitação por parte da criança.

Em relação ao respondedor, noutros estudos é também semelhante o predomínio da mãe. ${ }^{14-15}$ Pode dever-se ao facto de mesmo havendo mudanças pragmáticas na estrutura da família, a mãe continuar a ser considerada como a cuidadora principal. ${ }^{16}$

Relativamente aos tipos de aparelhos disponíveis, um estudo realizado em Singapura com 725 pais de 
TABELA 2. Fatores relacionados com o tempo de exposição de ecrã da criança (continuação)

Associação do tempo de ecrã com diferentes variáveis

\begin{tabular}{|c|c|c|c|c|c|}
\hline \multirow{2}{*}{$\begin{array}{l}\text { Variáveis } \\
\text { Número de aparelhos existentes/ } \\
\text { utilizados no quarto do filho }\end{array}$} & \multicolumn{2}{|c|}{ TEE em horas (semana) } & \multicolumn{2}{|c|}{ TEE em horas (fim-de-semana) } & \multirow[t]{2}{*}{ Teste estatístico } \\
\hline & & \multirow{5}{*}{$p=0,009 *$} & & \multirow{5}{*}{$p=0,011^{*}$} & \\
\hline 1 & $1,87( \pm 1,92)$ & & $2,27( \pm 2,03)$ & & \multirow{9}{*}{ ANOVA } \\
\hline 2 & $1,92( \pm 1,70)$ & & $2,14( \pm 1,64)$ & & \\
\hline 3 & $1,45( \pm 1.35)$ & & $2,80( \pm 3,03)$ & & \\
\hline 4 & $4,00( \pm 2,60)$ & & $6,33( \pm 2,31)$ & & \\
\hline Número de aparelho no quarto dos pais & & \multirow{5}{*}{$p=0,138$} & & \multirow{5}{*}{$p=0,218$} & \\
\hline 1 & $1,42( \pm 1,31)$ & & $1,89( \pm 1,81)$ & & \\
\hline 2 & $1,85( \pm 1,69)$ & & $2,22( \pm 1,99)$ & & \\
\hline 3 & $1,68( \pm 2,24)$ & & $1,43( \pm 1,72)$ & & \\
\hline 4 & $2,73( \pm 3,37)$ & & $1,87( \pm 1,49)$ & & \\
\hline Abordagem pelo médico & (mediana, AIQ) & \multirow{3}{*}{$p=0,304$} & (mediana, AIQ) & \multirow{3}{*}{$p=0,274$} & \multirow{6}{*}{ Mann-Whitney } \\
\hline Sim & $1,30(0,45-2,30)$ & & $1,30(0,33-2,50)$ & & \\
\hline Não & $1,30(0,45-2,0)$ & & $1,50(0,50-3,0)$ & & \\
\hline Abordagem pelo enfermeiro & & \multirow{3}{*}{$p=0,066$} & & \multirow{3}{*}{$p=0,268$} & \\
\hline Sim & $1,15(0,33-2,30)$ & & $1,15(0,30-2,45)$ & & \\
\hline Não & $1,30(0,50-2,0)$ & & $1,50(0,60-3,01)$ & & \\
\hline Tempo de ecrã dos pais (semana) & $r(s)=0,275$ & $p=0,01^{*}$ & & & \multirow{2}{*}{ Spearman } \\
\hline Tempo de ecrã dos pais (fim-de-semana) & & & $r(s)=0,448$ & $p=0,01^{*}$ & \\
\hline
\end{tabular}

Legenda:TEE = Tempo de exposição a ecrã; $p=$ Nível de significância; ${ }^{*}=$ Estatisticamente significativo; $r(s)=$ Coeficiente de correlação; AIQ = Amplitude interquartil; DP = Desvio-padrão.

crianças com idade $\leq 24$ meses revelou que a televisão $(44,8 \%)$ e o smartphone/tablet $(30,4 \%)$ foram os mais utilizados. ${ }^{17}$ Estes valores são sobreponíveis aos encontrados no estudo. Apesar de a televisão ser o aparelho digital mais utilizado, não sendo utilizado apenas por 3\% das crianças avaliadas, o tablet e o smartphone têm ganho terreno em relação às consolas de jogos. Pelo facto de serem portáteis e permitirem o acesso à Internet, estes aparelhos digitais facilitam o acesso a múltiplos conteúdos que podem ser inadequados, expondo as crianças de forma inesperada ou não intencional a material ofensivo, como conteúdo pornográfico, obsceno, racista ou violento, a práticas comerciais e publicitárias não-éticas ou contactos indevidos. ${ }^{1,18}$

Outro dos parâmetros avaliados foi a idade de início da exposição a aparelhos digitais, tendo-se verificado que mais de $90 \%$ das crianças inicia a utilização da te- levisão com idade inferior a 24 meses. Destas, cerca de $75 \%$ inicia antes dos 18 meses de idade. Este valor é semelhante ao obtido por um estudo português realizado ao nível de uma USF em ambiente urbano, com crianças dos cinco aos 17 anos, em que apenas $22 \%$ referiu ter começado a ver televisão depois dos 24 meses. ${ }^{19}$ Esta exposição precoce é preocupante, uma vez que até aos 24 meses de idade as crianças necessitam de interagir com os cuidadores e explorar o meio que as rodeia para adquirirem capacidades cognitivas, emocionais, motoras e de linguagem. ${ }^{7,10}$ Estes valores refletem ainda que os pais ou outros cuidadores privilegiam formas de entretenimento passivo, quando brincar de forma espontânea e centrada nas motivações da criança é fundamental para o seu desenvolvimento. ${ }^{1}$ Para além disso, um estudo coorte com 667 grávidas constatou que o TEE de uma criança com um ano de vida pode estar 
relacionado e servir de preditor do seu TEE aos dois e cinco anos. ${ }^{20}$

Em relação às circunstâncias em que são utilizados os aparelhos digitais e, avaliando a frequência do uso, verificou-se que em $22,7 \%$ da amostra os dispositivos são utilizados durante as refeições, praticamente todos os dias da semana. Do mesmo modo, o estudo TARGet Kids!, no Canadá, com crianças de três anos, verificou que em $31 \%, 11 \%$ e $17 \%$ dos casos a televisão estava ligada durante o pequeno-almoço, almoço e jantar, respetivamente. ${ }^{21}$ Em cerca de $19 \%$ dos casos, estes aparelhos são utilizados quase diariamente nos 60 minutos antes de dormir e para entreter a criança enquanto os pais fazem as tarefas do dia-a-dia. Um estudo português, ao nível dos cuidados de saúde primários, verificou que em crianças dos seis meses aos 10 anos, em idade pré-escolar, a prevalência de utilização da televisão para adormecer foi de $77 \%$. Este valor é claramente superior ao encontrado; contudo, pode estar sobrevalorizado pela amostra de dimensão reduzida $(n=56) .^{22}$ É importante perceber que a existência de atividade de teor excitante após o jantar é uma inadaptação, sendo preocupante que numa fase de estabelecimento do ciclo sono-vigília a televisão desempenhe um papel chave no ritual de adormecimento. ${ }^{22}$ Vários estudos mostraram que estes comportamentos parecem estar associados a alterações de hábitos alimentares e do sono, bem com na interação social e no aproveitamento escolar. ${ }^{1-4}$ Para além disso, a utilização excessiva de aparelhos digitais associa-se ao sedentarismo, por substituir outro tipo de atividades físicas e lúdicas. Num estudo americano verificou-se que por cada hora adicional de exposição à televisão, o risco de obesidade aumenta cerca de $2 \%$, associação que foi comprovada em dois outros estudos..$^{23-25}$

Outra situação específica de uso de aparelhos digitais, $o$ ato de premiar a criança, na amostra com 13,1\% ( $<1 \mathrm{vez} / \mathrm{semana}$ ) tem sido visto como algo eficaz no controlo do TEE. ${ }^{16}$ De facto, num estudo qualitativo com 53 pais de crianças dos 5-6 anos verificou-se que esta estratégia é também utilizada com caráter punitivo ou como método de negociação de atividades alternativas que não envolvem aparelhos digitais. ${ }^{26} \mathrm{Re}-$ lativamente ao uso de aparelhos digitais aquando de birra, um estudo numa população rural indiana, com crianças dos dois aos seis anos, verificou que $292 \mathrm{em}$
379 pais utilizavam estes aparelhos para acalmar os seus filhos. ${ }^{27}$ Esta proporção de $77 \%$ é superior aos $13,5 \%$ da amostra, o que poderá ser explicado por se tratar de uma população de um país em desenvolvimento, em que há mais dificuldade socio-emocional e menor autoperceção de controlo sobre o comportamento da criança. ${ }^{28}$ É fundamental combater esta ideologia de shut-up toy, parando com a promoção deste hábito que suprime a criança de criar os seus mecanismos internos de autorregulação perante situações adversas. ${ }^{29}$

Os aparelhos digitais mais presentes nos quartos das crianças avaliadas são a televisão e o tablet, com uma representatividade de $41 \%$ e $27 \%$, respetivamente. Estes dados estão de acordo com uma das situações mais frequentes de utilização: os 60 minutos antes de dormir. Num estudo americano, abrangendo crianças até aos seis anos, verificou-se que o aparelho digital mais frequentemente presente no quarto das crianças foi a televisão, nomeadamente em $18 \%$ até aos 24 meses, $43 \%$ dos três aos quatro anos e $37 \%$ a partir dos cinco anos. ${ }^{30}$ Este estudo apresenta ainda, como razões principais para os pais colocarem televisão no quarto das crianças, as seguintes: liberdade para verem os seus próprios programas; ocupação das crianças enquanto executam tarefas domésticas; ajuda no adormecimento das crianças. ${ }^{30}$ Vários autores apontam que o uso dos media pode afetar não só a quantidade total, mas também a qualidade do sono. Sabe-se que a visualização de conteúdos que envolvam suspense, drama e conflito podem ser demasiado emocionantes para as crianças e estão associadas a alterações fisiológicas como aumento das hormonas de stress, condicionando o atraso e má qualidade do sono. ${ }^{31}$ Para além disso, o uso destes aparelhos no quarto foi também associado a um maior risco de exposição excessiva, ${ }^{7}$ bem como a menor prática de atividade física. ${ }^{32}$ Neste sentido, a AAP recomenda a remoção da televisão e outros aparelhos digitais do quarto das crianças..$^{33-34}$ Neste estudo verificou-se uma diferença estatisticamente significativa entre o número de aparelhos digitais disponíveis no quarto e o TEE durante a semana $(p=0,009)$ e ao fim de semana $(p=0,011)$. Verificou-se também um aumento estatisticamente significativo do TEE numa criança com quatro aparelhos, comparativamente à que tem um aparelho $(p=0,003)$. Esta associação verificada 
noutro estudo, aumentando a prevalência de TEE $>2 \mathrm{~h}$ consoante se aumenta o número de aparelhos digitais disponíveis em casa, não se verifica significativa no ajuste do modelo de regressão. ${ }^{17}$ Por outro lado, o uso dos aparelhos digitais no quarto, um dos locais da casa mais isolados, propicia a visualização de conteúdos inapropriados, devido a uma menor supervisão dos pais ou cuidadores.

No presente estudo verificou-se que $25,2 \%$ das crianças vê televisão e $12,9 \%$ usa o smartphone praticamente todos os dias e sem supervisão de um adulto. Um questionário enviado a 1.977 pais australianos, em 2017, concluiu que aproximadamente $50 \%$ das crianças com menos de seis anos usavam aparelhos digitais sem supervisão adulta e que $20 \%$ o fariam cinco a sete vezes por semana, valor semelhante ao presente na amostra. ${ }^{35}$ Mais uma vez, verifica-se uma utilização não concordante com as recomendações da AAP e risco aumentado de exposição a conteúdos não adequados à idade. De acordo com as guidelines da AAP, é importante consciencializar os pais para a supervisão da utilização e procura de conteúdos adequados e de qualidade. ${ }^{7}$

Quando comparado o TEE das crianças com o dos pais ou cuidadores, é de referir que a média do TEE dos pais durante a semana foi de 4,40 horas $( \pm 4,05)$ e ao fim de semana foi de 3,42 horas $( \pm 3,40)$; por outro lado, nas crianças verificou-se que a média do TEE durante a semana foi de 1,66 horas $( \pm 1,65)$ e ao fim de semana foi de $2,04( \pm 1,95)$ horas. Verificou-se existir uma correlação positiva e estatisticamente significativa entre o TEE durante a semana dos pais e crianças $(r(s)=0,275 ; p<0,01)$ e do TEE ao fim de semana dos pais e crianças $(r(s)=0,448 ; p<0,01)$. As médias de TEE nas crianças foram sobreponíveis a outro estudo, $\operatorname{com} n=62$ e média de idade de 3,65 anos, mais especificamente 1,24 horas durante a semana e 1,88 horas ao fim de semana. ${ }^{16} \mathrm{~A}$ relação entre os TEE dos pais e crianças está bem estabelecida na literatura científica, defendendo uma revisão sistemática que o TEE dos pais é o fator mais frequentemente implicado e influente no TEE das crianças com idade pré-escolar. ${ }^{36} \mathrm{Um}$ estudo português revelou que crianças com pais ou cuidadores que assistam a mais de duas horas de televisão por dia tem um risco 1,5 a 8 vezes superior de uma exposição semelhante. ${ }^{37}$ Segundo a AAP, os pais com maior uso de aparelhos digitais têm menor interação verbal e não-verbal com os filhos, es- tando este associado a maior risco de conflito entre pais e filhos. Assim, sendo o TEE dos pais um bom preditor dos hábitos dos filhos, se houver uma redução do consumo por parte dos pais haverá um impacto positivo nos comportamentos adotados pelas crianças. ${ }^{7}$

Num estudo americano e num português menos de metade dos pais ou cuidadores inquiridos tinha conhecimento das recomendações existentes sobre o TEE adequado em crianças em idade pré-escolar. ${ }^{38-39}$ No presente estudo apenas $23,6 \%$ dos pais ou cuidadores reconhecem um uso exagerado dos media pelas crianças. No entanto, como já constatado anteriormente, a maior parte das crianças ultrapassa o TEE recomendado. Por outro lado, a maioria dos inquiridos, cerca de $76 \%$, refere que este tema nunca foi falado nas consultas de SIJ, mas que gostariam de o ver abordado. Este resultado provavelmente reflete a evidência que nos diz que os pais sentem dúvidas entre os efeitos positivos e negativos do TEE, nomeadamente em relação à saúde, expressando simultaneamente medo pela criança poder não ser capaz de lidar posteriormente com um quotidiano pautado por tecnologia. ${ }^{40}$ De facto, a educação para os media pode ajudar a reduzir o seu uso abusivo, tanto das crianças como dos seus pais ou cuidadores. Quanto mais informados estiverem os pais das crianças, mais capacitados estarão para uma melhor gestão do uso dos aparelhos digitais. ${ }^{11}$

No que diz respeito aos pais foi encontrada uma relação estatisticamente significativa entre a utilização de aparelhos digitais às refeições pelos pais e crianças $(p<0,0001)$, que é visto como um momento significante para o aumento do TEE nas crianças e como foco de potencial intervenção futura. ${ }^{16,21}$

Relativamente à perceção dos pais sobre o TEE da criança verificou-se uma diferença estatisticamente significativa $(p<0,0001)$ entre os valores médios de TEE durante a semana e fim de semana dos grupos «reduzido», «adequado» e «exagerado», mais especificamente com valores significativamente superiores de TEE quando a perceção dos pais designou o consumo «exagerado» ( $p<0,0001)$. Esta concordância é controversa na literatura e, por exemplo, um estudo com 80 crianças entre os quatro e os sete anos demonstrou que pode haver uma sobre ou subestimação do TEE das crianças pelos pais em três ou quatro horas, dependendo da presença da televisão no quarto da criança. ${ }^{41}$ 
Quanto à escolaridade do pai registou-se uma diferença estatisticamente significativa entre os valores médios do TEE para os diferentes graus. Contudo, essa diferença é única e estatisticamente significativa na comparação do grupo com escolaridade superior ao $12^{\circ}$ ano com o grupo com escolaridade equivalente ao $7^{\circ}-^{\circ}$ ano, em que o primeiro apresenta um TEE superior. Esta associação é controversa, com evidência a apontar no sentido de uma relação inversamente proporcional; contudo, um estudo coorte australiano com crianças de três a cinco anos mostra uma relação positiva. $^{14}$

De referir também o possível benefício da abordagem pelos profissionais de saúde, em que os papéis do médico e do enfermeiro permitiram médias de TEE inferiores, apesar de não atingirem a significância estatística. De facto, é um tópico recente, cuja integração não se encontra sistematizada na consulta de SIJ. Sabe-se, por exemplo, que apenas $16 \%$ dos pediatras iniciam uma discussão sobre este assunto. ${ }^{3}$ Assim sendo, é importante transmitir que não existe evidência de eficácia de nenhum tipo de intervenção breve sobre TEE em crianças. Contudo, o Royal College of Paediatrics and Child Health delineou quatro questões simples que podem ser incorporadas na história clínica e dão informação importante sobre o modus operandi da família -Is screen time in your household controlled?; Does screen use interfere with what your family want to do?; Does screen use interfere with sleep?; Are you able to control snacking during screen time? ${ }^{42}$ De forma sumária, são relevantes o controlo sobre duração de uso e conteúdos, a interferência do TEE na rotina da família e suas atividades, o impacto no ritual de adormecimento e o consumo de snacks durante a utilização destes aparelhos digitais. No que concerne ao aconselhamento existem algumas regras a transmitir aos pais durante a consulta, nomeadamente: definição de limites, quer em termos de TEE máximo por dia quer de áreas com proibição de uso de aparelhos digitais; a construção de um horário para a visualização de conteúdos em conjunto (pais e filhos); a listagem de tarefas/momentos da relação pais-filhos insubstituíveis e a delineação do que é uma etiqueta digital adequada e admitida pelos pais, da mesma forma que estes se têm de autodeterminar como um modelo confiável neste campo. ${ }^{43}$
De notar também que a televisão foi o aparelho mais transversal nas casas das famílias inquiridas, mas a sua presença nos quartos dos pais e crianças foi ainda mais reportada, com aproximadamente o dobro da frequência. Esta discrepância pode estar relacionada, empiricamente, com um possível destaque dos pais em relação aos smartphones e aos tablets por serem portáteis e mais «novidade», muitas vezes necessários em diferentes contextos que não o lúdico. Por outro lado, é conjeturável que quando o local referido é o quarto, e contrariamente à higiene do sono, a televisão tem um papel predominante, sendo o aparelho preferencial.

\section{CONCLUSÃO}

O presente estudo pretendeu demonstrar o incumprimento de normas internacionais relativamente ao limite diário estipulado para o TEE e, concomitantemente, perceber fatores que possam influenciar este mesmo TEE. Apesar de programas bem estabelecidos, como a RUA SÉSAMO ou as pistas da BluE, estarem relacionados com o desenvolvimento de capacidades em idade pré-escolar, as crianças com idade $<30$ meses necessitam de interação pessoal. E como foi demonstrado, o início do uso destes aparelhos digitais acontece muito frequentemente antes dos 18 meses. $\mathrm{O}$ aparecimento de alguns e-books com potencial de estimular a leitura precoce pela prática com letras, fonética e reconhecimento de palavras podem ao mesmo tempo ter atrações suficientes para distrair a atenção da criança da história e interferir com a compreensão da mesma.

Um dos efeitos mais negativos da televisão é a substituição dos momentos de interação de diálogo e brincadeiras com os pais. Há que não esquecer que a autorregulação, empatia, habilidades sociais e resolução de problemas são principalmente aprendidos através da exploração do ambiente natural, interagindo com colegas e cuidadores e jogando de forma desestruturada e criativa. Em crianças mais pequenas pode substituir atividades sensoriomotoras.

Assim, o médico e o enfermeiro de família assumem um papel primordial na promoção de ocupação ativa e prevenção das consequências sociais, emocionais e biológicas que estão associadas ao uso incorreto dos aparelhos digitais. 


\section{AGRADECIMENTOS}

Ao secretariado clínico de todas as USF envolvidas.

\section{COMISSÃO DE ÉTICA}

Estudo realizado após parecer favorável da Comissão de Ética para a Saúde da ARS Norte.

\section{REFERÊNCIAS BIBLIOGRÁFICAS}

1. Dias A, Almeida C, Lobo AL, Veiga G. Os média e as crianças lá de casa... [Media and children at home...]. Saúde Infantil. 2013;35(2):55-60. Portuguese

2. Rideout $\mathrm{V}$. Zero to eight: children's media use in America [homepage]. Common Sense Media; 2011. Available from: https://www.commonsensemedia.org/research/zero-to-eight-childrens-media-use-in-america-2011

3. Rideout V. Zero to eight: children's media use in America [homepage]. Common Sense Media; 2013. Available from: https://www.commonsensemedia.org/research/zero-to-eight-childrens-media-use-in-america-2013

4. Dias P, Brito R. Crianças ( 0 aos 8 anos) e tecnologias digitais: um estudo qualitativo exploratório - relatório nacional Portugal. Lisboa: Centro de Estudos de Comunicação e Cultura; 2016. ISBN 9789899928824

5. Kirkorian HL, Wartella EA, Anderson DR. Media and young children's learning. Future Child. 2008;18(1):39-61.

6. Ray M, Jat KR. Effect of electronic media on children. Indian Pediatr. 2010;47(7):561-8.

7. Council on Communications and Media. Media and young minds. Pediatrics. 2016;138(5):20162591.

8. Entidade Reguladora para a Comunicação Social. Crescendo entre ecrãs: usos de meios eletrónicos por crianças (3-8 anos) [homepage]. Lisboa: ERC; 2017. Available from: www.erc.pt/pt/estudos-e-publicacoes/consumos-de-media/estudo-crescendo-entre-ecras-usos-de-meios-eletronicos-por-criancas-3-8-anos

9. World Health Organization. Guidelines on physical activity, sedentary behavior and sleep for children under 5 years of age [homepage]. Geneva: WHO; 2019. Available from: https://apps.who.int/iris/handle/10665/325147

10. Guerra NG, Williamson AA, Lucas-Molina B. Normal development: infancy, childhood and adolescence. In: Rey JM, Martin A, editors. IACAPAP e-Textbook of child and adolescent mental health [Internet]. Geneva: International Association for Child and Adolescent Psychiatry and Allied Professions; 2015. chapter A.2. Available from: https://iacapap.org/content/uploads/A.2.-DEVELOPMENT-072012.pdf

11. von Elm E,Altman DG, Egger M, Pocock SJ, Gøtzsche PC, Vandenbroucke $J P$, et al. The Strengthening the Reporting of Observational Studies in Epidemiology (STROBE) statement: guidelines for reporting observational studies. J Clin Epidemiol. 2008;61(4):344-9.

12. Cheng S, Maeda T, Yoichi S, Yamagata Z, Tomiwa K. Early television exposure and children's behavioral and social outcomes at age 30 months. J Epidemiol. 2010;20 Suppl 2:S482-9.

13. Yeung WJ, Sandberg JF, Davis-Kean PE, Hofferth SL. Children's time with fathers in intact families. J Marriage Fam. 2001;63(1):136-54.

14. Downing KL, Hinkley T, Salmon J, Hnatiuk JA, Hesketh KD. Do the correlates of screen time and sedentary time differ in preschool children?
BMC Public Health. 2017;17:285.

15. Jago R, Sebire SJ, Edwards MJ, Thompson JL. Parental TV viewing, parental self-efficacy, media equipment and TV viewing among preschool children. Eur J Pediatr. 2013;172(11):1543-5.

16. Tang L, Darlington G, Ma DW, Haines J. Mothers' and fathers' media parenting practices associated with young children's screen-time: a cross-sectional study. BMC Obes. 2018;5:37.

17. Goh SN, Teh LH, Tay WR, Anantharaman S, van Dam RM, Tan CS, et al. Sociodemographic, home environment and parental influences on total and device-specific screen viewing in children aged 2 years and below: an observational study. BMJ Open. 2016;6(1):e009113.

18. Laranjeira C, Vidal F, Peres S. Televisão, videojogos e internet: hábitos de consumo [Television, videogames and internet: consumption habits]. Saúde Infantil. 2008;30(3):98-101. Portuguese

19. Figueiredo M, Sousa C, Teixeira C, Pinto F. Hábitos de exposição ao ecrã de uma população pediátrica de uma área urbana [Media exposure of a paediatric population in an urban health care centre]. Nascer Crescer. 2008;17(4):224-7. Portuguese

20. Xu H, Wen LM, Hardy LL, Rissel C. A 5-year longitudinal analysis of modifiable predictors for outdoor play and screen-time of 2- to 5-yearolds. Int J Behav Nutr Phys Act. 2016;13(1):96.

21. Birken CS, Maguire J, Mekky M, Manlhiot C, Beck CE, Jacobson S, et al. Parental factors associated with screen time in pre-school children in primary-care practice: a TARGet Kids! study. Public Health Nutr. 2011;14(12):2134-8.

22. Pedrosa C, Cruz G, Pereira SA. Hábitos e perturbações do sono de uma população infantil de Vila Nova de Gaia [Sleep practices and disorders in a paediatric population of Vila Nova de Gaia]. Acta Pediatr Port. 2004;35(4):323-8. Portuguese

23. Dietz JrWH, Gortmaker SL. Do we fatten our children at the television set? Obesity and television viewing in children and adolescents. Pediatrics. 1985;75(5):807-12.

24. Kuriyan R, Bhat S, Thomas T, Vaz M, Kurpad AV. Television viewing and sleep are associated with overweight among urban and semi-urban South Indian children. Nutr J. 2007;6:25.

25. Zimmerman FJ. Children's media use and sleep problems: issues and unanswered questions [Internet]. Kaiser Family Foundation; 2008. Available from: https://www.kff.org/wp-content/uploads/2013/01/7674 .pdf

26. Jago R, Zahra J, Edwards MJ, Kesten JM, Solomon-Moore E, Thompson $\mathrm{J}$, et al. Managing the screen-viewing behaviours of children aged 56 years: a qualitative analysis of parental strategies. BMJ Open. 2016;6(3):e010355.

27. Shah RR, Fahey NM, Soni AV, Phatak AG, Nimbalkar SM. Screen time usage among preschoolers aged 2-6 in rural Western India: a cross-sectional study. J Family Med Prim Care. 2019;8(6):1999-2002.

28. Radesky JS, Peacock-Chambers E, Zuckerman B, Silverstein M. Use of mobile technology to calm upset children: associations with socialemotional development. JAMA Pediatr. 2016;170(4):397-9.

29. Radesky JS, Schumacher J, Zuckerman B. Mobile and interactive media use by young children: the good, the bad, and the unknown. Pediatrics. 2015;135(1):1-3.

30. Vandewater EA, Rideout VJ, Wartella EA, Huang X, Lee JH, Shim M. Digital childhood: electronic media and technology use among infants, 
toddlers, and preschoolers. Pediatrics. 2007;119(5):e1006-15.

31. Robinson TN. Television viewing and childhood obesity. Pediatr Clin North Am. 2001;48(4):1017-25.

32. Adachi-Mejia AM, Longacre MR, Gibson JJ, Beach ML, Titus-Ernstoff LT, Dalton MA. Children with a TV in their bedroom at higher risk for being overweight. Int J Obes. 2007;31(4):644-51.

33. StrasburgerVC, Council on Communications and Media, American Academy of Pediatrics. Media education. Pediatrics. 2010;126(5):1012-7.

34. Council on Communications and Media, Brown A. Media use by children younger than 2 years. Pediatrics. 2011;128(5):1040-5.

35. Rhodes A. Screen time and kids: what's happening in our homes? [Internet]. Australian Child Health Poll; 2017. Available from: https://www.rchpoll.org.au/wp-content/uploads/2017/06/ACHPPoll7_Detailed-Report-June21.pdf

36. Xu H,Wen LM, Rissel C. Associations of parental influences with physical activity and screen time among young children: a systematic review. J Obes. 2015;2015:546925.

37. Jago R, Stamatakis E, Gama A, Carvalhal IM, Nogueira H, Rosado V, et al. Parent and child screen-viewing time and home media environment. Am J Prev Med. 2012;43(2):150-8.

38. Funk JB, Brouwer J, Curtiss K, McBroom E. Parents of preschoolers: expert media recommendations and ratings knowledge, media-effects beliefs, and monitoring practices. Pediatrics. 2009;123(3):981-8.

39. Patraquim C, Ferreira S, Martins H, Mourão H, Gomes P, Martins S. As crianças e a exposição aos media [Children and media exposure]. Nascer Crescer. 2018;27(1):11-21. Portuguese
40. Solomon-Moore E, Matthews J, ReidT, Toumpakari Z, Sebire SJ, Thompson JL, et al. Examining the challenges posed to parents by the contemporary screen environments of children: a qualitative investigation. BMC Pediatr. 2018;18(1):129.

41. Robinson JL, Winiewicz DD, Fuerch JH, Roemmich JN, Epstein LH. Relationship between parental estimate and an objective measure of child television watching. Int J Behav Nutr Phys Act. 2006;3:43.

42. Viner R, Davie M, Firth A. The health impacts of screen time: a guide for clinicians and parents [Internet]. The Royal College of Paediatrics and Child Health; 2019. Available from: https://www.rcpch.ac.uk/sites/default/files/2018-12/rcpch_screen_time_guide_-_final.pdf

43. Shifrin D, Brown A, Hill D, Jana L, Flinn SK. Growing up digital: media research symposium [Internet]. American Academy of Paediatrics; 2015. Available from: https://www.aap.org/en-us/Documents/digital_media_symposium_proceedings.pdf

\section{CONFLITO DE INTERESSES}

Os autores declaram não ter quaisquer conflitos de interesse.

\section{ENDEREÇO PARA CORRESPONDÊNCIA}

Nuno Miguel Parente

E-mail: nunomiguelpm@gmail.com

https://orcid.org/0000-0002-4198-243X

Recebido em 03-10-2019

Aceite para publicação em 14-03-2020

\section{ABSTRACT}

\section{USAGE OF MEDIA BY CHILDREN FROM 12 MONTHS TO 5 YEARS OF AGE}

Objective: To characterize the use of media by preschool children and to compare this use with AAP and WHO recommendations. Test associations between children's use of digital devices and certain parental characteristics and their use.

Type of study: Cross-sectional, descriptive, and analytical.

Location: Family Health Units (FHU) from the northern region of Portugal.

Population: Users aged 12 months to 5 years of six FHU.

Methods: Anonymous questionnaire was applied to a sample of parents who accompanied the child to the consultation. It included demographic variables and questions related to the use of media by children and parents.

Results: We obtained 342 questionnaires. The television and smartphone were the most frequently used devices in the households of the surveyed families and the ones most frequently used by children. The use began before 18 months in $74.7 \%$ for television, $23.4 \%$ for smartphone and $16.3 \%$ for tablet. The daily use of television and smartphone was $65.9 \%$ and $24 \%$, respectively. 19\% reported the use of the devices in the 60 minutes before sleep and the 'use during mealtime' was the main reason addressed by parents. In children aged $<24$ months, 88-93\% demonstrated using media; from children aged > 24 months, 70-74\% had a screen time $>1 \mathrm{~h} /$ day. An association was established between children's screen time and parental screen time, parents' perception of their child's media use, and the number of media devices available in the bedroom.

Conclusions: There is a very early onset, before 18 months, for the use of these technologies, which seems to be abusive, worrisome, and outside of the current guideline standards. It is important to address this issue early in consultations, encouraging parents to other types of activity and stimulation.

Keywords: Screening time; Guidelines; Behavior; Children; Parents. 


\section{ANEXO I \\ Questionário \\ Nota introdutória ao questionário}

\section{Introdução Questionário:}

A utilização de aparelhos digitais (computador, telemóvel, consolas de jogos, tablets, etc.) faz parte da rotina do dia-a-dia de qualquer família. Vários estudos comprovam que estas tecnologias influenciam os comportamentos das crianças e, como tal, a sua correta utilização é um desafio a alcançar.

A realização deste trabalho tem como objetivo identificar e caracterizar a utilização destes aparelhos por crianças dos 12 meses aos 5 anos de idade, bem como a atitude dos pais face a este tema.

PARTE I. Identificação

CRIANÇA:

Género: $\square$ Rapaz $\square$ Rapariga

Idade:

$\square$ Menos 18 meses

18 aos 24 meses(incluído)

Mais de 24 meses

Durante a maioria do tempo em que está acordada, a criança fica aos cuidados de:

$\square$ Pais $\square$ Avós $\square$ Ama
$\square$ Infantário/creche/pré-escola? $\square$ Outros?
$\square$ (Quem?

MÃE:

Idade:

19 anos ou menos

25-29 anos

20-24 anos

35 anos ou mais

30-34 anos

Escolaridade:

$\square \quad 1^{\circ}$ ao 4 ano

$\square \quad 7^{\circ}$ ao $9^{\circ}$ ano

Mais de $12^{\circ}$ ano

PAI:

Idade:

19 anos ou menos

20-24 anos

25-29 anos

30-34 anos

$5^{\circ}$ ao $6^{\circ}$ ano

$10^{\circ}$ ao $12^{\circ}$ ano
Assim, pede-se aos pais de crianças com 12 meses a 5 anos de idade que preencham o seguinte questionário. Demorará cerca de 5 minutos a preenchê-lo. Este é anónimo e confidencial.

Se já preencheu este questionário previamente, por favor, não o faça novamente!

Ao preencher o seguinte questionário aceita participar neste estudo e os dados obtidos serão única e exclusivamente utilizados para este estudo.

Quando terminar, por favor, coloque o inquérito na caixa devidamente identificada para o efeito. Obrigado!

Escolaridade:
$1^{\circ}$ ao 4 ano
$5^{\circ}$ ao $6^{\circ}$ ano
$7^{\circ}$ ao $9^{\circ}$ ano
$10^{\circ}$ ao $12^{\circ}$ ano
Mais de $12^{\circ}$ ano
QUEM ESTÁ A RESPONDER AO QUESTIONÁRIO?
Pai $\square$ Mãe

PARTE II. As perguntas seguintes referem-se ao uso de aparelhos digitais pela criança.

1. Assinale com um $(X)$ os aparelhos digitais que existem em sua casa? (pode assinalar mais do que um)

Televisão

Computador (fixo ou portátil)

Consola de jogos (ligada TV ou portátil)

Tablet

Telemóvel/Smartphone

Outro?

2. Assinale com um (X) os aparelhos digitais que a criança usa habitualmente? (pode assinalar mais do que um)

Televisão

Computador (fixo ou portátil)

Consola de jogos (ligada TV ou portátil)

Tablet

Telemóvel/Smartphone

Outro?

35 anos ou mais 
3. Com que idade a criança começou a usar os seguintes aparelhos digitais:

\begin{tabular}{|l|l|l|l|l|}
\hline & Antes dos 18 meses & Entre os 18 meses e os 24 meses (inclusive) & Depois dos 24 meses & Nunca usou \\
\hline Televisão & & & & \\
\hline Tablet & & & & \\
\hline Telemóvel/Smartphone & & & & \\
\hline Computador (fixo ou portátil) & & & & \\
\hline Consola de jogos (ligada TV ou portátil) & & & & \\
\hline Outro? & & & \\
\hline
\end{tabular}

4. Assinale com um $(X)$ com que frequência a sua criança usa os seguintes aparelhos digitais?

\begin{tabular}{|l|l|l|l|l|l|}
\hline & $5 x$ a 7x por semana & $3 x$ a $4 x$ por semana & $1 x$ a 2x por semana & Menos 1x por semana & Nunca usou \\
\hline Televisão & & & & & \\
\hline Tablet & & & & & \\
\hline Telemóvel/Smartphone & & & & & \\
\hline Computador (fixo ou portátil) & & & & & \\
\hline Consola de jogos (ligada TV ou portátil) & & & & & \\
\hline Outro? & & & & \\
\hline
\end{tabular}

5. Num dia típico de semana, quanto tempo em média a criança usa cada um dos seguintes aparelhos digitais? (por exemplo, 15 min. ou 1 hora e 30 minutos)

\begin{tabular}{|c|c|c|c|}
\hline Televisão & $\mathrm{h}: \ldots$ min & Computador (fixo ou portátil) & $\mathrm{h}: \ldots$ min \\
\hline Tablet & $\mathrm{h}: \ldots$ min & Consola de jogos (ligada TV ou portátil) & $\mathrm{h}: \ldots \min$ \\
\hline Telemóvel/Smartphone & $\mathrm{h}: \ldots \min$ & Outro? & $\mathrm{h}: \ldots \min$ \\
\hline
\end{tabular}

6. Num dia de fim-de-semana, quanto tempo em média a criança usa cada um dos seguintes aparelhos digitais? (por exemplo, 15 min. ou 1 hora e 30 minutos)

\begin{tabular}{|c|c|c|c|}
\hline Televisão & $\ldots \mathrm{h}: \ldots \min$ & Computador (fixo ou portátil) & $\mathrm{h}: \ldots \min$ \\
\hline Tablet & $\ldots \mathrm{h}: \ldots \min$ & Consola de jogos (ligada TV ou portátil) & $\mathrm{h}: \ldots$ min \\
\hline Telemóvel/Smartphone & $\mathrm{h}: \ldots$ min & Outro? & $\mathrm{h}: \ldots \min$ \\
\hline
\end{tabular}

7. Com que frequência a criança usa aparelhos digitais nas seguintes situações?

\begin{tabular}{|l|l|l|l|l|l|}
\hline & $5 x$ a 7x por semana & $3 x$ a 4x por semana & 1x a 2x por semana & Menos 1x por semana & Nunca usou \\
\hline Durante as refeições & & & & & \\
\hline Nos 60 minutos antes de dormir & & & & \\
\hline $\begin{array}{l}\text { Entreter a criança enquanto os pais } \\
\text { fazem as tarefas do dia-a-dia/trabalham }\end{array}$ & & & & \\
\hline Distrair a criança quando ela faz birra & & & & \\
\hline $\begin{array}{l}\text { Entreter a criança nas reuniões com } \\
\text { família/amigos }\end{array}$ & & & & \\
\hline Premiar a criança & & & & \\
\hline Outro? & & & & \\
\hline
\end{tabular}


8. Assinale com um (X) os aparelhos digitais que existem no quarto da criança ou que ela usa no quarto? (pode assinar mais do que um)

$\square$ Televisão

$\square$ Computador (fixo ou portátil)
Consola de jogos (ligada TV ou portátil)

Tablet

Telemóvel/Smartphone

Outro?

9. Assinale com um $(X)$ com que frequência a sua criança usa os seguintes aparelhos digitais sozinha?

\begin{tabular}{|l|l|l|l|l|l|}
\hline & $5 x$ a 7x por semana & $3 x$ a 4x por semana & 1x a 2x por semana & Menos 1x por semana & Nunca usou \\
\hline Televisão & & & & & \\
\hline Tablet & & & & & \\
\hline Telemóvel/Smartphone & & & & & \\
\hline Computador (fixo ou portátil) & & & & & \\
\hline Consola de jogos (ligada TV ou portátil) & & & & & \\
\hline Outro? & & & & & \\
\hline
\end{tabular}

10. Na sua opinião, o uso dos aparelhos digitais pela criança é:
$\square$ Reduzido
$\square$ Adequado
$\square$ Exagerado

11. Alguma vez estes profissionais de saúde falaram consigo sobre a utilização dos aparelhos digitais pela criança?

$\checkmark$ Médico de Família $\square$ SIM $\square$ NÃO

$\checkmark$ Enfermeiro de Família $\square$ SIM $\square$ NÃO

12. Gostaria que o médico de família e/ou enfermeiro de família falasse consigo sobre o uso dos aparelhos digitais?

$\square$ SIM

$\square \quad$ NÃO
PARTE III. As perguntas seguintes referem-se ao uso de aparelhos digitais por si.

1. Assinale com um (X) os aparelhos digitais que usa habitualmente? (pode assinalar mais do que um)

$\square$ Televisão

$\square$ Computador (fixo ou portátil)

$\square$ Consola de jogos (ligada TV ou portátil)

$\square$ Tablet

$\square$ Telemóvel/Smartphone

Outro?

2. Assinale com um $(X)$ com que frequência usa os seguintes aparelhos digitais?

\begin{tabular}{|l|l|l|l|l|l|}
\hline & $5 x$ a 7x por semana & $3 x$ a 4x por semana & $1 x$ a 2x por semana & Menos 1x por semana & Nunca usou \\
\hline Televisão & & & & & \\
\hline Tablet & & & & & \\
\hline Telemóvel/Smartphone & & & & \\
\hline Computador (fixo ou portátil) & & & & & \\
\hline Consola de jogos (ligada TV ou portátil) & & & & & \\
\hline Outro? & & & & & \\
\hline
\end{tabular}

3. Num dia típico de semana, quanto tempo em média usa cada um dos seguintes aparelhos digitais? (por exemplo, 15 min. ou 1 hora e 30 minutos)

\begin{tabular}{|l|c|l|l|}
\hline Televisão & $\mathrm{h}: \_$min & Computador (fixo ou portátil) & $\ldots \mathrm{h}: \_\mathrm{min}$ \\
\hline Tablet & $\mathrm{h}: \_$min & Consola de jogos (ligada TV ou portátil) & $\ldots \mathrm{h}: \_$min \\
\hline Telemóvel/Smartphone & $\mathrm{h}: \_$min & Outro? & $\ldots$ \\
\hline
\end{tabular}


4. Num dia de fim-de-semana, quanto tempo em média usa cada um dos seguintes aparelhos digitais? (por exemplo, 15 min. ou 1 hora e 30 minutos)

\begin{tabular}{|c|c|c|c|}
\hline Televisão & $\mathrm{h}: \ldots \min$ & Computador (fixo ou portátil) & $\mathrm{h}: \ldots \min$ \\
\hline Tablet & $\mathrm{h}: \ldots \min$ & Consola de jogos (ligada TV ou portátil) & $\mathrm{h}: \ldots \min$ \\
\hline Telemóvel/Smartphone & $\mathrm{h}: \ldots \min$ & Outro? & $\mathrm{h}: \ldots \min$ \\
\hline
\end{tabular}

5. Com que frequência usa os aparelhos digitais durante as refeições?

$\square \quad 5 x$ a $7 x$ por semana

$\square \quad 3 x$ a $4 x$ por semana

$\square \quad 1 x$ a $2 x$ por semana

Menos de $1 x$ por semana

Nunca usou
6. Assinale com um $(X)$ os aparelhos digitais que existem ou usa no seu quarto? (pode assinar mais do que um) Televisão

$\square$ Computador (fixo ou portátil)

$\square$ Consola de jogos (ligada TV ou portátil)

$\square$ Tablet

$\square$ Telemóvel/Smartphone

Outro? 